\title{
The unlikely matters
}

\author{
The study of cosmic impacts and the effects they have offers two lessons for students of science.
}

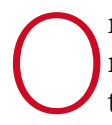
n 15 June, a telescope near Tucson, Arizona, picked up a relatively fast-moving dot in the sky: an asteroid of the sort the telescope is designed to seek. Now known as 2008 LV16, this small rock's orbit takes it from well out beyond Mars to almost as far sunward as Venus, passing by Earth on the way. LV16 poses no threat to anyone, and probably never will. But there is always a chance that, way down the line, something will get in the way of its wanderings. If that obstruction is the Earth, LV16 will cause an explosion on a par with an all-out nuclear exchange between superpowers.

What is remarkable about LV16 is not the risk it poses; over the next century that risk is negligible. It is that the risk has been recognized and quantified - as it has for many, if not yet all, potentially hazardous asteroids. As Alan Harris shows in a Commentary on page 1178, part of this week's special package of material on cosmic impacts, the simple expedient of finding the larger near-Earth asteroids and analysing their orbits has reduced the best estimate of the danger they pose to human life by about $90 \%$.

When cataloguing near-Earth asteroids was first discussed in the 1980s, much of the astronomy community was non-plussed, and some of it was actively hostile. A fair sample of these bodies' orbits, it was argued, is all a scientist needs to see: listing them all would be stamp-collecting, not science. And from a strictly scientific perspective, this had some merit. But as a response to a real, if rather goofy-sounding, threat, it was not enough. The creation of an exhaustive catalogue was the right thing to do.

This illustrates a more general principle: the public-interest response to a problem uncovered by scientific research is not necessarily to do more scientific research. Something more structured and pragmatic may be appropriate, even if it is less likely to bring academic kudos. When the public funds research, it expects, when the need arises, that scientists will use their knowledge and tools for more than just their own scientific interests, even if that

EDITORIAL
1143 The unlikely matters
NEWS FEATURES
$1157 \quad \begin{aligned} & \text { Tunguska at } 100 \\ & \text { Duncan Steel }\end{aligned}$

1160 The hole at the bottom of the Moon Eric Hand

1164 The burger bar that saved the world David Chandler

1170 All craters great and small

\section{COMMENTARY}

1178 What Spaceguard did

Alan Harris

BOOKS \& ARTS

1184 In retrospect: Lucifer's Hammer

Oliver Morton

1185 Message from the heavens Martin Kemp

\section{NEWS \& VIEWS}

1191 Forming the martian great divide Walter S. Kiefer

\section{LETTERS}

1212 The Borealis basin and the martian crustal dichotomy Jeffery C. Andrews-Hanna et al.

1216 Mega-impact formation of the Mars hemispheric dichotomy Margarita M. Marinova et al.

1220 Implications of an impact origin for the Martian hemispheric dichotomy Francis Nimmo et al.

For podcast, video material and more, see www.nature.com/news/specials/cosmicimpacts/index.html. an unspotted asteroid laying waste to a significant chunk of land, as happened in the Tunguska region of Siberia 100 years ago this week; and imagine if that area, unlike Tunguska and a surprising amount of the globe today, were populated. The politician or scientific adviser who had dismissed such a disaster as being too improbable to bother with would be in dire straits. Politicians know in their bones that unlikely events matter.

And that is the second lesson that the study of cosmic impacts teaches. Although impacts follow statistical patterns, individual events and their quirks can have unpredictable effects. As various researchers report in this issue, an impact more than four billion years ago shaped Mars's geological history by dividing its surface into highlands and lowlands, providing a sink in which the planet's water, such as it may have, could pool. An even larger impact gave Earth its Moon. And yet another, at the end of the Cretaceous period, removed Earth's remaining dinosaurs, among a great many other life-forms. Thoughts of impacts have, since the 1980 paper in which Luis Alvarez and his son Walter and their colleagues first detailed that end-Cretaceous event, re-established the possibility of catastrophic change in the scientific imagination, even when the crisis has nothing to do with asteroids or comets.

The Universe is lumpy, irregular, surprising, dramatic. The better this is understood, the better the interplay of chance and necessity can be appreciated. And that same understanding can, more pragmatically, form the basis for vigilance, for planning and for action. - 